\title{
Strengths Based Talent Development Centre
}

\author{
Llewellyn van Zyl $\left.\right|^{1}$ \\ 1 Eindhoven University of Technology
}

The Strengths Based Talent Development Centre is a hybrid strengths-based capability assessment and development intervention which aims to identify and develop talentreadiness and leader capability, bench streng th for key positions, person-environment fit, potential and capacity for upward mobility into more complex roles. Specifically, its purpose is to (a) assess and develop individual capability, (b) provide real-time people analytics aligned to the org anisational strategy and (c) to aid talent mapping, career pathing and succession planning. 